\title{
Recurrence and Pain after Mesh Repair of Inguinal Hernias
}

Wyckliffe Kaisha Otsianyi, Joseph Wang'ombe Githaiga

School of Medicine, University of Nairobi

Correspondence to: Dr. Wyckliffe Kaisha Otsianyi, P.0 Box 368-00202, Nairobi. Email: wyksa2000@uonbi.ac.ke

\begin{abstract}
Background: Surgery for inguinal hernias has evolved over a prolonged period of time with the main outcome of interest being recurrence and pain. Mesh hernioplasty has been practiced with increasing frequency at Kenyatta National hospital (KNH) since its formal introduction in 2006. There has not been any published data on outcome of hernia treatment at this institution for both non-mesh and mesh techniques. Objective: To evaluate the rates of recurrence and inguinodynia at $\mathrm{KNH}$ for patients undergoing mesh repair. Methods: The study was conducted on all inguinal hernia patients operated between 1st October 2006 and 30th October 2011. Demographic and treatment characteristics were abstracted from
\end{abstract}

\section{Introduction}

Inguinal hernia repair is the most frequent operation in general surgery (1). The most important criteria for the choice of surgical technique are safety, recurrence rate and satisfaction to the patient (2). Recurrence rates vary considerably for the various techniques: from less than $5 \%$ to $25 \%$ (3). Recurrences are important as many of these need repeated surgery (4). Repeated surgeries are difficult with predictable increased morbidity and overall costs. Improving the results would therefore have a useful medical and economic impact. Mesh techniques have been reported to have the lowest recurrence rates, with Lichtenstein as the prototype (5). In the recent past, a newer technique that combines the three principles of onlay, plug and underlay mesh placement has been developed. Such meshes include the prolene hernia system (PHS) or ultrapro hernia system (UHS). This bilayer mesh has shown promising results. Overall, there was a trend toward decreased complications, with a significant difference in the hematoma/seroma rates, and a significant decrease in the recurrence files. The outcomes of interest (recurrence and inguinodynia) were obtained from follow up data from patients' files or by telephone survey where these were missing or were incomplete. Results: A total of 181 patients had complete demographic and operative data in their files over the study period. Of these 129 were followed for a mean duration of 37 months. The hernia recurrence rate was $4.7 \%$ while the inguinodynia rate was $30.2 \%$. Conclusion: There is a low recurrence rate but a high prevalence of inguinal pain and discomfort (inguinodynia).

Keywords: Hernia, Inguinodynia, Recurrence, Mesh

Ann Afr Surg. 2016;13(2): 56-8.

DOI: http://dx.doi.org/10.4314/aas.v13i2.5

rate for the PHS mesh group $(6,7)$. Inguinodynia is pain or discomfort lasting greater than 3 months after surgery (8). However, it is suggested that it may be due to entrapment of the ilioinguinal, iliohypogastric or genital branch of the genitofemoral nerve either in the sutures, mesh or scar tissue (9-11). The aim of this study was to document the recurrence and inguinodynia rates among patients undergoing mesh hernioplasty at $\mathrm{KNH}$, a non-hernia specialist centre.

\section{Methods}

After study approval by institutional ethical board, data from eligible patients were abstracted from the patients' records. All patients who had been operated for groin hernia using mesh technique at KNH since year 2006 were included in the study. Demographic and peri-operative information was collected. The files were further scrutinized for two outcomes of interest (hernia recurrence and pain/ discomfort). To increase completeness of the data, phone calls were made to the patients to inquire if hernia had recurred and if they had inguinodynia. Two trained final year 
medical students were utilized to make the calls and every tenth call was corroborated by the main author. The respondents were asked if a swelling similar to a previous one had recurred and if they had discomfort or pain in the groin that interfered with their activities of daily living.

\section{Results}

A total of 246 (250 herniae) patients met the eligibility criteria and were included in the study. The mean age was 44.1 years ( SD 17.7, range 16-85). The modal age group was 30-39 years. Male patients were the majority at $95.1 \%(n=234)$. Majority of the hernias were right sided ( $\mathrm{n}=160,65.3 \%)$ with4 hernias being bilateral (1.6\%). Only $101(40 \%)$ of the hernia were classified with indirect (lateral) forming $71 \%$ of them. Majority ( $\mathrm{N}=181,73.6 \%)$ were operated using mesh technique utilizing Lichtenstein $(73,40.3 \%)$ and bilayer $(104,57.5 \%)$. Four of the mesh techniques were not defined. Micro-porous mesh (prolene type) was utilized in $126(70 \%)$ of the cases, while macroporous vypro in $44(24.4 \%)$ and ultrapro in 3 cases.

Of the 181 patients with mesh repair, 129 were traced via telephone for assessment of pain and recurrence. The follow up period ranged from 14 to 91 months with a median follow up period of 37 months (3 years 1 month). Six patients reported a recurrence representing $4.7 \%$ over the follow up period. Of these, 3 had a bilayer device inserted while the other 3 had a Lichtenstein repair. Thirty nine (30.2\%) reported pain or discomfort in the groin over the same follow up period. Of these, 24 had a bilayer repair, while the other 15 had a Lichtenstein repair.

\section{Discussion}

Inguinal hernia is a common surgical condition that is highly prevalent among men (12). It is associated with risk of various complications including obstruction, strangulation and potential for death. Untreated, even when life threatening complications do not occur, it may enlarge and interfere with the individuals' quality of life (13). When no contraindications exist, inguinal hernias have continued to be treated by surgery. However, it has continued to be dogged by multiple complications, amongst them being a high recurrence rate (3). Other complications include seroma, infection and persistent groin pain (inguinodynia). Pain can be quite debilitating and influence the choice of a treatment modality (14). The treatment of hernia has a large economic impact, given the high prevalence of the disease. The impact of treatment of a recurrence is higher. Surgery for recurrent disease is more difficult with potential for higher morbidity (15). In a metaanalysis recurrence rates reduced from 3.6\% vs. $0.8 \%$ when Shouldice technique "the gold standard" was compared with mesh repair $(16,17)$. These rates differ from specialist versus non specialist hernia centers. Our non-specialist centre had a recurrence rate of $4.7 \%$. Burcharth in a review of the Danish hernia registry reported an overall inguinal hernia reoperation rate of $3.8 \%$. He acknowledged re-operation rates underestimate recurrence rates (18). Other studies have reported rates that do not widely differ from ours (19).

Post-surgical chronic pain represents a major, largely unrecognized clinical problem. High rates of pain have been reported. Several studies and randomized clinical trials indicate that up to $30 \%$ of patients report some form of pain 1 year after Lichtenstein hernia repair $(20,21)$. Our rate of inguinal pain or inguinal discomfort was similarly quite high at $30.2 \%$. A recent meta-analysis suggested that use of preperitoneal approach and mesh placement may have less prevalence of pain due to reduced dissection that affects nerves in the inguinal canal (22).

\section{Conclusion}

The inguinal hernia recurrence rates reported are acceptable in a non-hernia specialist center. However, like other authors the pain and groin discomfort rates were high. We suggest that pain should be the new target of outcome measure given the low rates of recurrence.

\section{References}

1. Rutkow IM. Demographic and Socioeconomic Aspects of Hernia Repair in the United States in 2003. SurgClin North Am. 2003;83(5):1045-51.

2. Badkur M, Garg N. Comparative Study of Prolene Hernia System and Lichtenstein Method for Open Inguinal Hernia Repair. J Clin Diagn Res. 2015; 9(6): PC04-PC07.

3. Grant AM. Open Mesh Versus Non-Mesh Repair of Groin Hernia: Meta-Analysis of Randomised Trials Based on Individual Patient Data. EU Hernia Trialists Collaboration. Hernia. 2002; 6(3):130-6.

4. Nilsson E, Anderberg B, Bragmark M., et al. Hernia Surgery in a Defined Population. Improvements Possible in Outcome and Cost-Effectiveness. Ambul Surg.1993; 1(3): 150-3.

5. Nordin P, Bartelmess P, Jansson C, et al. Randomized Trial of Lichtenstein Versus Shouldice Hernia Repair in General Surgical Practice. Br J Surg. 2002; 89:(1): 45-9.

6. Awad SS, Yallampalli S, Srour AM, et al. Improved Outcomes with the Prolene Hernia System Mesh Compared with the Time-Honored Lichtenstein Onlay Mesh Repair for Inguinal Hernia Repair. Am J Surg. 2007; 193(6): 697-701. 
7. Huang CS, Huang CC, Lien HH. Prolene Hernia System Compared with Mesh Plug Technique: A Prospective Study of Short-to Mid-Term Outcomes in Primary Groin Hernia Repair. Hernia. 2005; 9(2): 167-171.

8. Hakeem A, Shanmugam V. Inguinodynia Following Lichtenstein Tension-Free Hernia Repair: A Review. World J Gastroenterol. 2011; 17(14):1791-6.

9. Starling JR, Harms BA, Schroeder ME, et al. Diagnosis and Treatment of Genitofemoral and Ilioinguinal Entrapment Neuralgia. Surgery. 1987; 102(4):581-6.

10. WantzGE. Testicular Atrophy and Chronic Residual Neuralgia as Risks of Inguinal Hernioplasty. SurgClin North Am. 1993; 73(3):571-81.

11. Racz G, Hagstrom D. Iliohypogastric and Ilioinguinal Nerve Entrapment: Diagnosis and Treatment. Pain Digest. 1992; 2:43-8.

12. Primatesta P, Goldacre MJ. Inguinal Hernia Repair: Incidence of Elective and Emergency Surgery, Readmission and Mortality. Int J Epidemiol. 1996; 25(4):835-9.

13. Mathonnet $M$, Mehinto D. Indications for Inguinal Hernia Repair. J Chir (Paris). 2007; 144 Spec No 4:5S11-4.

14. Poobalan S, Bruce J, King P, et al. Chronic Pain and Quality of Life Following Open Inguinal Hernia Repair. Br J Surg. 2001; 88(8): 1122-6.

15. Sevonius D. Recurrent Groin Hernia-Outcome after Surgery. Diss. Lund University, 2014.***

16. Scott NW, McCormack K, Graham P, et al. Open Mesh Versus Non-Mesh for Groin Hernia Repair. Cochrane Database Syst Rev. 2002;(4):CD002197.

17. Amato B, Moja L, Panico S, et al. Shouldice Technique versus Other Open Techniques for Inguinal Hernia Repair. Cochrane Database Syst Rev. 2012;(4):CD001543.

18. Burcharth J. The Epidemiology and Risk Factors for Recurrence after Inguinal Hernia Surgery. Dan Med J. 2014; 61(5): B4846.

19. Neumayer L, Giobbie-Hurder A, Jonasson O, et al. Open Mesh Versus Laparoscopic Mesh Repair of Inguinal Hernia. N Engl J Med. 2004;350(18):1819-27.

20. Bay-Nielsen M, Kehlet H, Strand L, et al. Quality Assessment of 26,304 Herniorrhaphies in Denmark: A Prospective Nationwide Study. Lancet. 2001; 358(9288):1124-8.

21. O'Dwyer PJ, Kingsnorth AN, Molloy RG, et al. Randomized Clinical Trial Assessing Impact of a Lightweight or Heavyweight Mesh on Chronic Pain after Inguinal Hernia Repair. Br J Surg. 2005; 92(2):166-70.

22. Willaert W, De Bacquer D, Rogiers X, et al. Open Preperitoneal Techniques Versus Lichtenstein Repair for Elective Inguinal Hernias. Cochrane Database Syst Rev. 2012;(7):CD008034. 